Review

\title{
Surface and bulk characterisation of electrospun membranes: Problems and improvements
}

\author{
David R. Nisbet ${ }^{a}$, Andrew E. Rodda ${ }^{a}$, David I. Finkelstein ${ }^{b}$, Malcolm K. Horne ${ }^{c}$, \\ John S. Forsythe ${ }^{\mathrm{a}}$, Wei Shen ${ }^{\mathrm{d}, *}$ \\ a CRC for Polymers, Department of Materials Engineering, Monash University, PO Box 69M, Victoria 3800, Australia \\ b The Mental Health Research Institute of Victoria, 155 Oak Street, Parkville, Victoria 3052, Australia \\ ${ }^{c}$ Howard Florey Institute, Gate 11, Royal Parade, The University of Melbourne, Victoria 3010, Australia \\ ${ }^{\mathrm{d}}$ Australian Pulp and Paper Institute, Department of Chemical Engineering, Monash University, PO Box 69M, Victoria 3800, Australia
}

\section{A R T I C L E I N F O}

\section{Article history:}

Received 27 October 2008

Received in revised form 28 January 2009

Accepted 30 January 2009

Available online 27 February 2009

\section{Keywords:}

Electrospinning

Nano-fibre

Characterisation

Membrane

Fibre

Mechanical properties

Surface properties

\begin{abstract}
A B S T R A C T
Electrospun membranes are used in a variety of applications, including filtration systems and sensors for chemical detection, and have attracted increased interest in the field of tissue engineering and regenerative medicine. Successful integration of these materials into a specific technology will require understanding of the fibres' surface, bulk and architectural properties. Detailed characterisation of these properties is frequently overlooked, particularly in specialised interdisciplinary fields such as tissue engineering. In this article we have reviewed the current status of the characterisation of electrospun membranes, while recommending improvements in using these techniques to better understand these very interesting nanostructured materials.
\end{abstract}

Crown Copyright $\odot 2009$ Published by Elsevier B.V. All rights reserved.

\section{Contents}

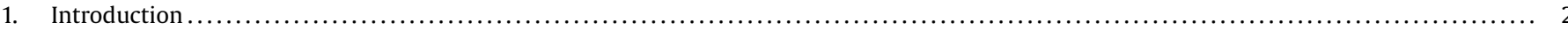

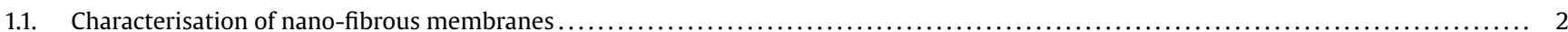

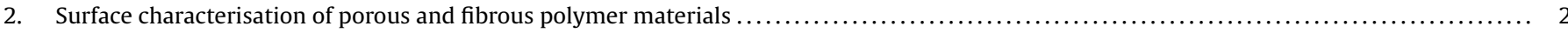

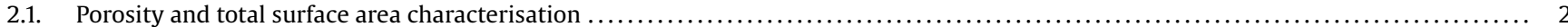

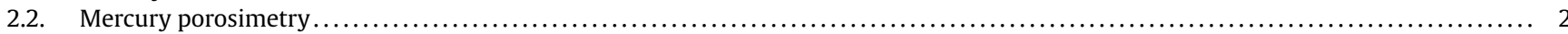

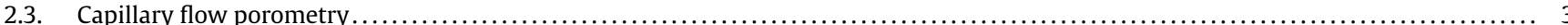

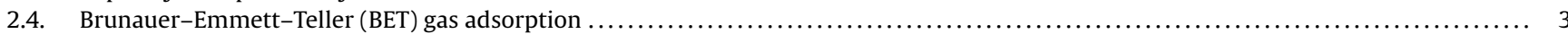

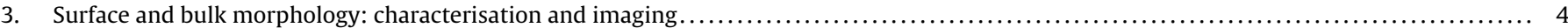

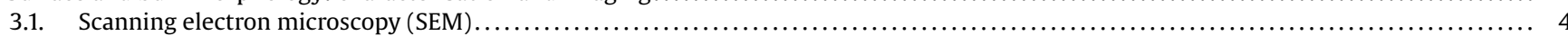

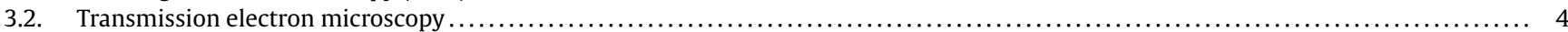

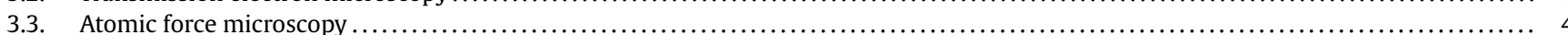

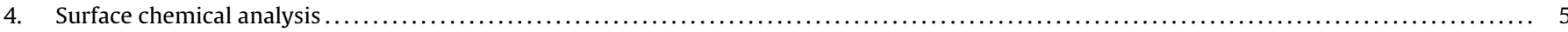

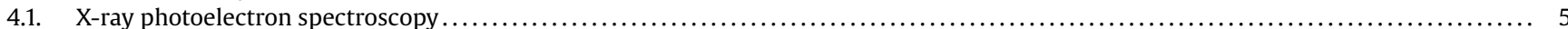

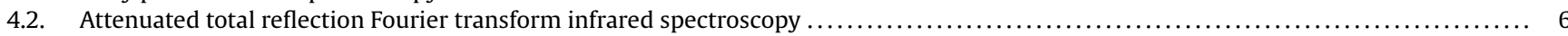

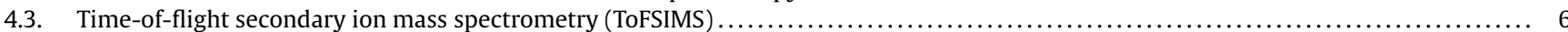

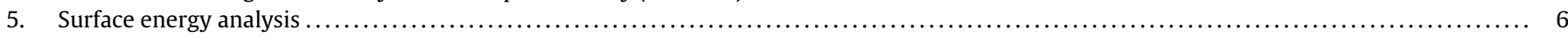

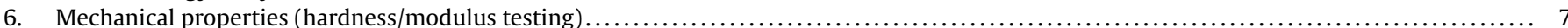

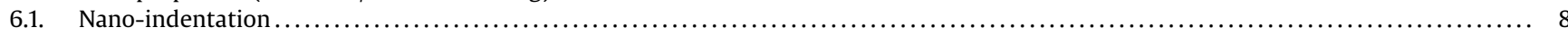

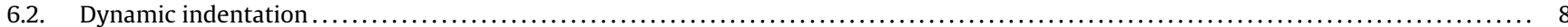

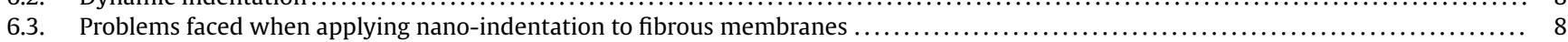

\footnotetext{
* Corresponding author. Tel.: +61 39905 3447; fax: +61 399053413.

E-mail address: wei.shen@eng.monash.edu.au (W. Shen).
} 


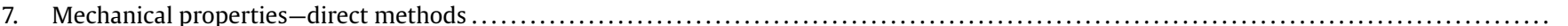

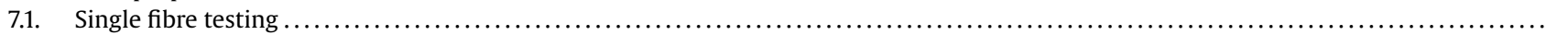

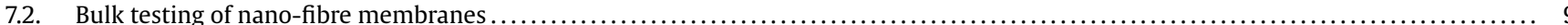

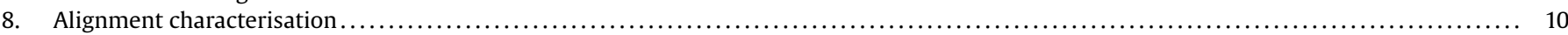

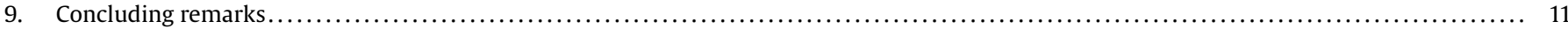

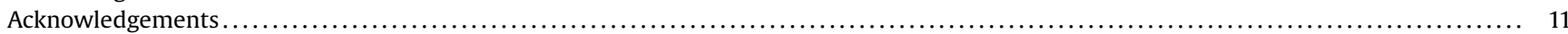

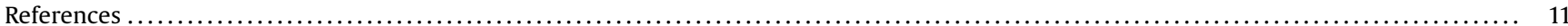

\section{Introduction}

As more advanced materials are synthesised and fabricated, their characterisation by surface science becomes both more difficult and more relevant [1]. Characterisation by surface science is paramount to understanding and generating advanced, functional and "smart" materials in modern technologies such as metallurgy, microelectronics, nano-technology, corrosion and tissue engineering. This review focuses on the surface characterisation of non-woven polymer membranes that are produced by electrospinning. Many of the same principles would however be relevant to fibrous membranes fabricated using other processes. We have also addressed the characterisation of modulus and architectural properties of such membranes.

The surface properties of polymeric materials strongly influence their interactions with other materials/interfaces within their surrounding environment and therefore determine the polymeric materials' suitability for particular engineering applications. The main surface properties of polymeric materials are (a) geometric and topographic and (b) surface chemical properties. Geometric and topographic properties include the roughness of two-dimensional polymer surfaces [2-4], porosity [5,6], pore size [2,7-9], pore size distribution [7,10], the specific surface area for porous and non-woven polymer membranes, and intra-fibre surface roughness [11]. Surface chemical properties and chemical composition can be different from that of the bulk [12] and in some instances can be altered by surface modification $[5,13]$. This can affect water wettability/hydrophilicity $[3,5,6,14]$ and surface energetics $[15,16]$.

\subsection{Characterisation of nano-fibrous membranes}

Although the technology for fabricating electrospun nanofibres was first reported in 1934 [17], the growing demand for nanostructured materials and their unique properties has led to renewed interest in electrospinning, particularly for tissue engineering and regenerative medical technologies. This includes nanostructured drug delivery vessels, cellular microenvironments and conduits for cell regeneration after injury or disease.

In such interdisciplinary fields, biologists will frequently overlook the effect that material surfaces will have on the biological questions they are asking [18], while material and surface scientists may see biological questions as too complex for the analytical tools at their disposal. This gap in the perspective of different fields can be bridged by assembling interdisciplinary teams, and through the emergence of "new" scientists whose expertise crosses the traditional fields of materials engineering, surface science and biology. This review is a contribution to bridging this gap and addresses the current status of the characterisation of electrospun membranes, highlighting the advantages and disadvantages of analytical techniques in different applications. Where possible, we have recommended improvements in the choice and application of techniques used for characterisation, in the hope of providing a more informed choice of characterisation methods.

\section{Surface characterisation of porous and fibrous polymer materials}

\subsection{Porosity and total surface area characterisation}

Electrospun membranes are highly porous (Fig. 1) and therefore have very large surface-to-volume ratios, a property central to their use in technologies such as filtration $[19,20]$, chemical sensing [21] and tissue engineering [3,9,22]. Consequently, accurate characterisation of these membranes is required to optimise their use in such fields. One simple method for determining porosity of electrospun membranes is by measuring their apparent weight to volume ratio with the knowledge of the bulk polymer density [20]. This results in a very basic description of porosity. Other techniques, including mercury porosimetry, capillary flow porosimetry and Brunauer-Emmett-Teller (BET) gas adsorption allow for a far more detailed characterisation.

\subsection{Mercury porosimetry}

Mercury porosimetry is a traditional method for characterising porosity and pore size distribution $[10,16]$. It is a liquid intrusion method, which relies on the principle behind the Laplace equation. Mercury has a high surface tension $\left(485.5 \mathrm{dyn} / \mathrm{cm}\right.$ at $\left.25^{\circ} \mathrm{C}\right)$ [23] and therefore forms large contact angles with most other materials $\left(\cong 130^{\circ}\right)$ [24]. Based on the Laplace equation [23], mercury does not spontaneously penetrate pores by capillary action, and therefore an external pressure must be applied to force mercury into the pores. By assuming that the pores in porous materials are cylindrical in shape, the pore diameters intruded by mercury can be correlated with the applied pressure according to the following equation [24]:

$D=\frac{-4 \gamma \cos \theta}{p}$

where $D$ is the diameter of the pore, $\gamma$ is the surface tension of mercury, $\theta$ is the contact angle between mercury and the solid and $p$ is the applied pressure. The direct data acquired is the accumulated volume of mercury entering the porous system. A pore size distribution curve is obtained by relating the log differential intrusion volume to $\log$ pore diameter, i.e., $d V / d(\log D)$. A description of this procedure can be found in Ref. [23].

Assuming pores are cylindrical, the pore surface area can also be calculated from the accumulated mercury intrusion volume and the pore diameter data. Since the surface area of a cylindrical pore is $\pi D L$ and the volume of the pore is $\pi D^{2} L / 4$ (where $D$ and $L$ are the diameter and the length of the cylindrical pore), the wall area of the pore thus equals to:

$A=\frac{4 V}{D}$

Ryu et al. [25] found that mercury porosimetry could detect changes in pore sizes of electrospun nylon-6 membranes of different fibre diameters. Instead of relying on the pore size data obtained directly from the log differential intrusion curve, they calculated the average pore diameters using Eq. (2) with the intrusion volume and pore surface area data obtained by BET measurements (discussed later). Their pore diameter results ranged from 2.737 to $0.167 \mu \mathrm{m}$ 

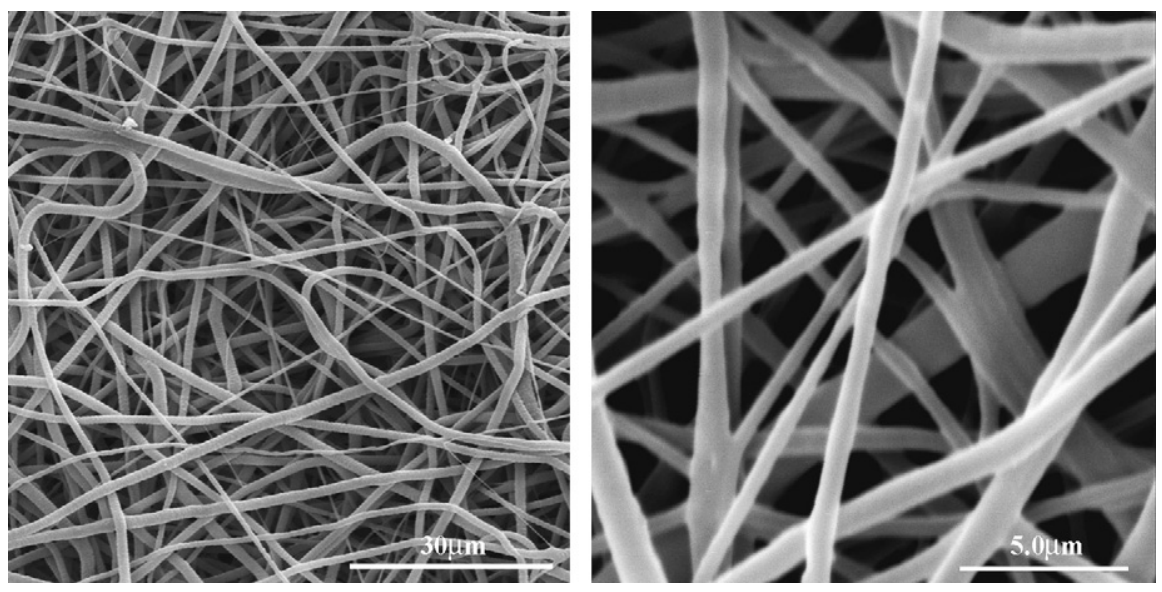

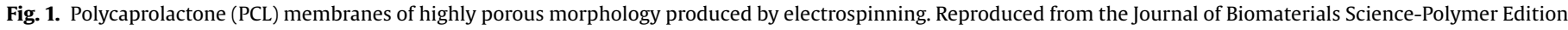
with permission from BRILL [22].

[25]. Direct volumes of mercury intrusion also provided data on the fraction of voids within the membrane, which ranged from 25 to $80 \%[25]$.

Unfortunately, assuming that pores are cylindrical is often an over-simplification. The use of Eq. (2) may not provide an accurate surface area measurement, due to a well-known problem associated with the so-called "bottle neck" effect. This occurs where large cavities exist behind narrow necks, which are considered to be pores having the diameter of the neck [23]. Furthermore, high pressure applied to polymer membranes has been reported to cause irreversible structural compression [26]. Thus the mercury intrusion method can only provide useful representations of porosity and surface area $[11,23,25]$ providing the above caveats are considered.

\subsection{Capillary flow porometry}

Capillary flow porometry is a liquid extrusion method for measuring pore size [27]. It requires that all pores first be completely filled with a wetting liquid, which is then forced out of the pores by a non-reacting gas. The work done by the gas in forcing liquid out of the pores is assumed to be equal to the surface free energy when the sample-liquid interface is replaced by the sample-gas interface.

$p d V=\left(\gamma_{s / g}-\gamma_{s / l}\right) d S$

where $p$ is the pressure applied; $d V$ is the displaced volume of liquid by gas; $\gamma_{s / g}$ and $\gamma_{s / l}$ are the surface free energies of solid-gas and solid-liquid; $d S$ is the increase of solid-gas surface area. Jena and Gupta [27] proposed that, by combining Eq. (3) with the Young's equation (Eq. (4)), and by replacing the pore cross-section area with the equal area circle, a working equation (Eq. (5)) could be derived:

$\gamma_{s / g}-\gamma_{s / l}=\gamma_{l / g} \cos \theta$

$p=\frac{4 \gamma_{l / g} \cos \theta}{D}$

where $\gamma_{l / g}$ is the liquid gas interfacial tension or liquid surface tension; $D$ is the diameter of the equivalent circular area of the pore cross-section [27].

Capillary flow porometry has been used to study the pore size distribution of electrospun fibre membranes $[8,28]$. Since capillary flow porometry is a liquid extrusion method, it is more appropriate to use the receding contact angle, which is usually small, causing $\cos \theta$ in Eq. (5) to approach unity. Capillary flow porometry is suitable for measuring "through pores" that connect one side of the material to the other side, but is not suitable for closed (isolated pores in the matrix) or blind-end pores (connected to the external surface via a single channel or orifice) [28]. Like mercury porosimetry, capillary flow porometry is also sensitive to the bottle neck effect [28], but unlike mercury porosimetry can not measure blind-end pores.

\subsection{Brunauer-Emmett-Teller (BET) gas adsorption}

BET gas adsorption method is a well-established method for characterising surface area and pore structure of porous materials. The fundamental consideration is the quantity of a single molecular adsorption layer of a known gas. By assuming the Langmuir adsorption model and incorporating the concept of multimolecular layer adsorption, as well as knowing the area occupied by a single adsorbed molecule, the surface area of the substrate can be calculated [23]. Gregg and Sing [29] have presented theoretical details of the method. The BET equation is usually written in the following form to allow the determination of values of $V_{m}$ and $C$ by graphical means [29]:

$\frac{P}{V_{a}\left(P_{0}-P\right)}=\frac{1}{V_{m} C}+\frac{C-1}{V_{m} C}\left(\frac{P}{P_{0}}\right)$

where $P$ and $P_{0}$ are the gas pressure and the saturated gas pressure at the temperature of experiment, $V_{a}$ and $V_{m}$ are the quantity of gas adsorbed under pressure $P$ and the quantity of gas required for a monolayer adsorption on the sample surface, respectively. $C$ is a constant related to the heat of adsorption of the first molecular layer of gas. By plotting $P / V_{a}\left(P_{0}-P\right)$ against $\left(P / P_{0}\right) . C$ and $V_{m}$ can be calculated from the intercept and the slope of the straight line [29]. Since there is no assumption on cylindrical pore geometry, the specific surface area determined by the BET method is more reliable than capillary flow and mercury porosimetry.

Ryu et al. used an image analyser to measure the diameters of electrospun fibres (which were controlled by the concentration of polymer solutions and ranged between 90 and $480 \mathrm{~nm}$ ), and found using BET that the total pore area of nano-fibrous membranes to range between 9 and $51 \mathrm{~m}^{2} / \mathrm{g}$, with the surface area decreasing for an increasing fibre diameter [25]. Based on their specific surface area and porosity data, the authors assumed that pores within the non-woven nano-fibrous membranes were fully interconnected when using this method. Although the experimental specific surface area data of nano-fibre membrane was smaller, it did not differ greatly from the value calculated using Eq. (2). The small diameter of the fibres, rather than fibre surface morphology, were found to contribute the most to the large specific surface areas found in this study [25]. 


\section{Surface and bulk morphology: characterisation and imaging}

\subsection{Scanning electron microscopy (SEM)}

Surface morphology is a critical parameter that can not only affect the responses of secondary surfaces (such as cells in tissue engineering) [9,22], but can also affect the method of characterisation for the materials surface [3]. SEM is a method that provides qualitative assessment of fibre diameter distribution, surface roughness and architecture of electrospun polymer membranes $[3,11,14,30]$. Because this method is very well understood and already extensively used, a detailed discussion will not be provided here. However, care must be taken to ensure that fibre diameters and interfibre spacings measured using SEM, are taken on the image plane to ensure that the scale is correct for postanalysis. More quantitative evaluation of surface roughness may be obtained using atomic force microscopy (for nano-scale roughness) and a range of contact and non-contact (optical) profilometers (for larger scale roughnesses) [31].

\subsection{Transmission electron microscopy}

Transmission electron microscopy (TEM) is a characterisation method where the beam of electrons is transmitted through a thin sample and the interaction of the electrons and the sample are used to generate an image. This method has been used on electrospun membranes to image core-shell structures (shown in Fig. 2 [32]) and electrospun fibres with incorporated composite particles [33] such as nano-tubes [34]. Like SEM, this method is well understood and is extensively used to image electrospun membranes, so a detailed discussion of the method is not warranted. In general TEM is a good method for imaging the inclusion of nano-domains within electrospun fibres. One potential problem with this method is the size of the samples that can be imaged, as they must be ultrathin (less than $1 \mu \mathrm{m}$ ) to allow the focus beam to pass through the sample. However this is rarely an issue for electrospun fibres, as most have a diameter smaller than this. They must also be able to withstand high vacuum.

\subsection{Atomic force microscopy}

Atomic force microscopy (AFM) was originally developed to probe the topography of surfaces [35], and has been used for this

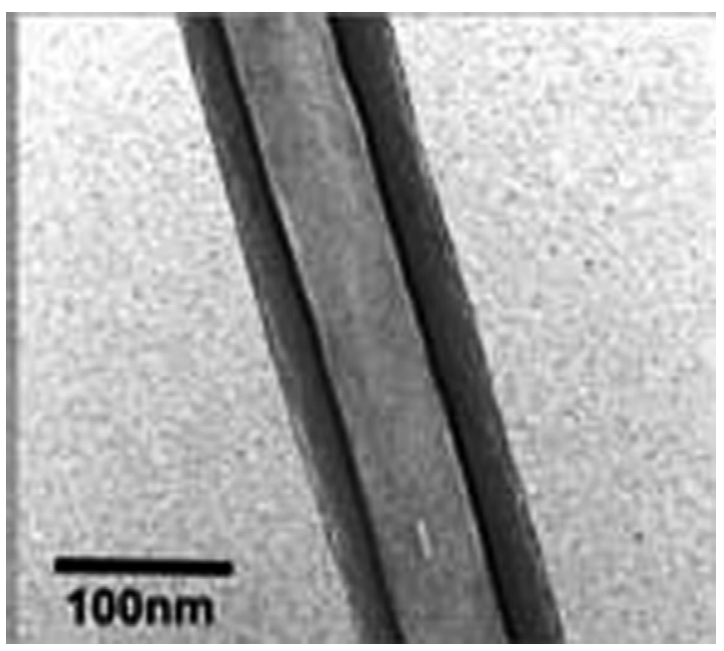

Fig. 2. TEM image of a hollow or core-shell electrospun polymer fibre produced from poly(vinylpyrrolidone). Reproduced from Microfluid Nanofluid with permission from Springer-Verlag [32].
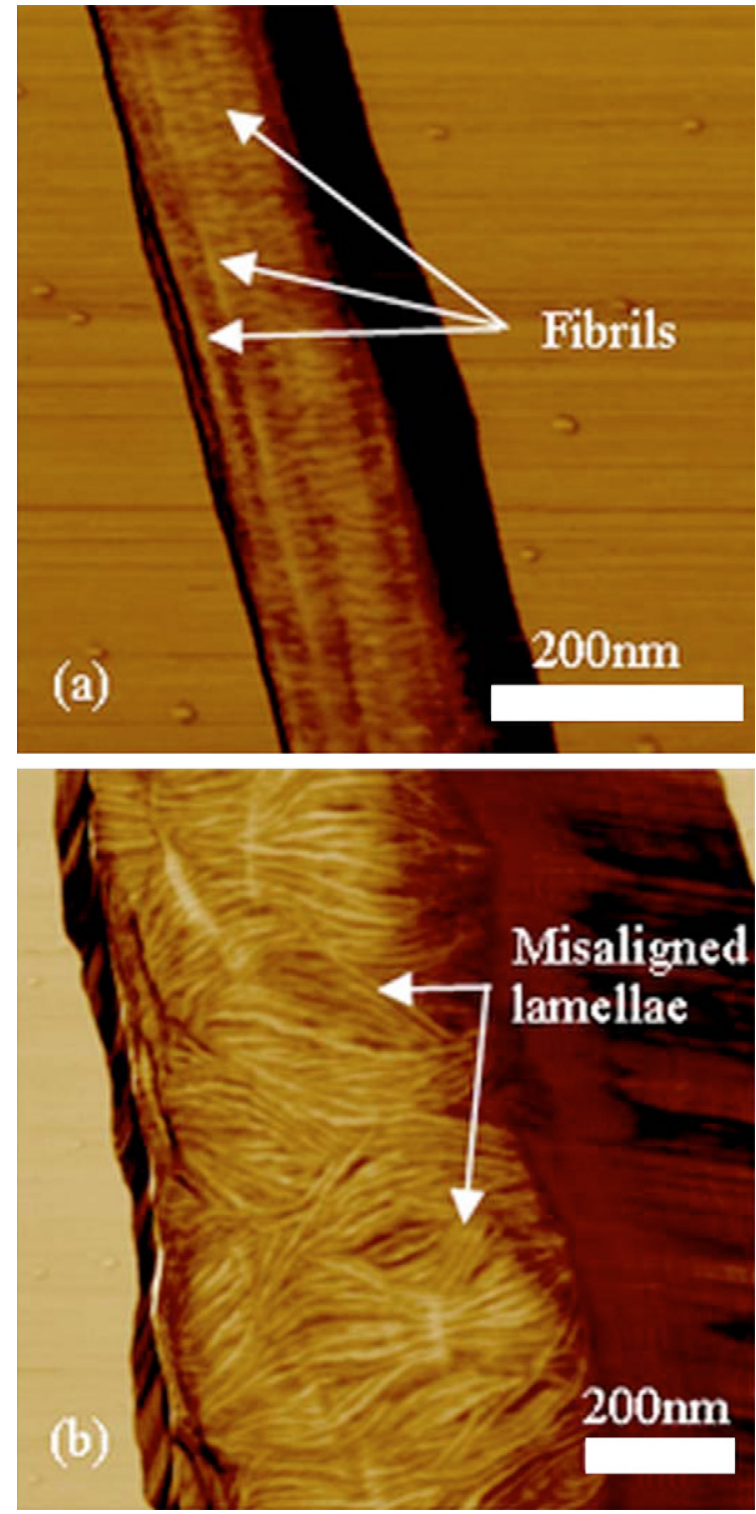

Fig. 3. AFM phase images highlighting the surface morphology of electrospun PCL nano-fibres. The fibril structure consists of alternating layers of crystalline and amorphous tie molecules and the lamellae structure most likely consists of relaxed amorphous tie molecules. As can be seen, extremely high resolutions can be obtained using this technique [36]. Reproduced from Applied Physics Letters with permission from AIP Journals.

purpose on polymer samples, including electrospun membranes (Fig. 3) [36], for many years. Several innovations have allowed the study of other surface properties, such as the distribution of a chemical of interest in blended mixtures and the distribution of crystalline and amorphous areas on a polymer surface, and these may have future applications in the characterisation of nano-fibres. The hardness and elastic modulus (through nano-indentation or more recent micromanipulation techniques) can also be determined using this equipment, and this will be examined in the "Mechanical Properties" section of this review. Thus AFM is a very flexible tool for characterising electrospun membranes and indeed any nano-scale surface.

Contact mode is the most conceptually simple form of AFM imaging and is analogous to feeling a surface's texture by running fingers over it. However this technique is generally unsuitable for electrospun polymers, as even soft cantilevers have stiffnesses comparable or even higher than the stiffness of the bonds in many polymers. Attempts at imaging will therefore cause damage to the 
sample, due to lateral (frictional) forces [37]. Contact mode has been used [38] to investigate adhesion force on electrospun PET synthetic fibres after the surfaces were modified by plasma treatment. The interested reader is referred to the following excellent review for a more detailed discussion of contact mode AFM imaging [35]. Non-contact modes also exist, which use an oscillating AFM tip to investigate the attractive force near the surface. This method has the advantage of causing less structural damage to the sample but is currently only rarely used for nano-fibre imaging [39].

The AFM mode most often used for polymer imaging and indeed the characterisation of electrospun membranes is "amplitude modulation", "intermittent contact" or "tapping" mode. This mode has allowed imaging of fibres (see Fig. 3) with a much higher degree of resolution than would be possible using SEM [36]. The tip is driven at a fixed frequency, allowing both the amplitude and phase change of the oscillation to be monitored. The amplitude will vary according to the strength of the force interaction between the surface and the tip, and can be used as negative feedback to control the height of the AFM tip. As the name "tapping" suggests, the tip only briefly enters the contact zone during measurement. Tapping mode generates smaller forces than contact modes, an important factor when imaging electrospun polymers. Vertical forces are reduced because the tip barely taps the surface, while lateral forces are minimised, as the tip is not in constant contact with the surface during scanning. This dramatically reduces surface damage to polymer samples [35]. It is also possible to perform AFM in a liquid medium, further reducing required forces [35,37], although the tip response can be more difficult to analyse in such an environment. The advantages of tapping mode (with phase imaging, discussed below) have made it the standard for imaging polymers and characterising electrospun membranes.

Phase imaging, where the image is created by comparing the phase of the cantilever excitation with the phase of the response oscillation, has allowed many different properties of polymer fibres to be investigated. Phase imaging has been used to detect fine detail on surfaces that also contain larger variations in height, but can also be used to detect a change in energy dissipation [37] of the material at the surface. This has allowed the detection of composition variation in blended polymer materials, even when topography does not change $[38,40]$, which may be useful in the future study of blended electrospun membranes or even electrospun core-shell structures. Some studies have been able to discern differences between crystalline and amorphous areas of a polymer sample based on the phase image, which has recently been applied to a study of the crystal structure of nano-fibres [36].

It should be noted that phase imaging cannot directly test the chemical makeup of the sample; all of the above techniques rely on changes in mechanical response between the different chemical moieties or phases. Thus limited contrast between properties of materials, for example hardness, limits their detection by phase imaging. Attempts at directly sensing both the physical and chemical makeup of a nano-scale sample have been made by combining AFM with both IR [41] and soft X-ray spectroscopy [42].

Notwithstanding the power of AFM in surface analysis, users must be wary of its limitations when analysing images of electrospun membranes. AFM images do suffer from small amounts of distortion, in both horizontal and vertical directions. Horizontal images tend to be broadened when a non-ideal tip interacts with the surface. For example, AFM most likely overestimates the diameters of individual electrospun fibres because of the AFM tip geometry [43], and while the correct choice of tip and a filtering algorithm may reduce this phenomenon, it can never be eradicated. Vertical images tend to be compressed by the downward forces exerted on the surface, although with appropriate selection of operating conditions this distortion is usually quite small $(0.1 \mathrm{~nm})$ and is unlikely to be a major problem for most polymer-based imaging.
Surface roughness is often measured by AFM and can be expressed in many ways, but is most commonly stated in terms of area average roughness $\left(R_{a}\right)$ and area root-mean-square roughness $\left(R_{m s}\right)$.

$R_{a}=\frac{1}{N} \sum_{i=1}^{N}\left|Z_{i}-\bar{Z}\right|$

$R_{m s}=\sqrt{\frac{1}{N} \sum_{i=1}^{N}\left\langle Z_{i}-\bar{Z}\right\rangle^{2}}$

where $N$ is the total number of data acquired, $\left(Z_{i}-\bar{Z}\right)$ is the height $(z$-value) difference between each data point and the average $z$ value of all data points. Most AFM software also calculates actual scan area $A_{\text {actual }}$ as a ratio of the projected scan area $A_{\text {proj }}$ [3], which is a useful index of roughness when calculating the surface energy of the substrate (discussed later in this review).

Providing these limitations of AFM are understood and acknowledged, and the correct operating conditions are used, AFM is very useful technique for high-resolution imaging of nano-fibres.

\section{Surface chemical analysis}

Techniques for chemical investigation of porous polymeric surfaces include X-ray photoelectron spectroscopy (XPS, also known as electron spectroscopy for chemical analysis or ESCA), Fourier transform infrared spectroscopy (FTIR) and time of flight secondary ion mass spectrometry (ToF-SIMS). When using these techniques, their application to electrospun membranes is similar to a $2 \mathrm{D}$ film, which is widely understood, hence only a brief description of their use for the surface chemical characterisation of electrospun membranes is warranted.

\subsection{X-ray photoelectron spectroscopy}

The most commonly used surface chemical analysis technique for polymers and biomedical materials, including electrospun membranes, is XPS $[5,12-14,30,44]$. It has been extensively reviewed as an experimental tool for biomaterial research and is a very sensitive surface technique [45]. The main reason for this is that the average mean-free-path of photoelectrons of the relevant kinetic energy range is small; meaning that the sampling depth is therefore localised to around $10 \mathrm{~nm}$ below the surface [44]. It has been reported that XPS is capable of a detection limit for protein adsorption of as low as $10 \mathrm{ng} / \mathrm{cm}^{2}$ at the near surface [46], highlighting its great sensitivity.

XPS uses soft X-ray sources, i.e., $\mathrm{Mg} \mathrm{K \alpha}$ or $\mathrm{Al} \mathrm{K} \alpha$ lines to eject photoelectrons from sample surfaces. It relies on binding energy shifts of photoelectrons ejected from the inner shells of atoms to identify their chemical difference and bonding environment [47]. As spectral resolution is most affected by the energy width of the incident X-ray, it can be improved by use of monochromators [47]. The binding energy shifts of $\mathrm{C} 1 \mathrm{~s}, \mathrm{O} 1 \mathrm{~s}$ and $\mathrm{N} 1 \mathrm{~s}$ have been widely used to derive information of functional groups on surfaces of biomaterials [46-48]. XPS has also been used to investigate whether full encasement has occurred in studies of electrospun polymer membranes with a core-shell structure [49,50] and other similar coatings [51]. However, the binding energy shifts can sometimes be too small to allow full characterisation of the surface; curve fitting must then be used to de-convolute XP spectra. Modern XP spectrometers provide computer software for curve fitting using multivariable analysis methods.

Synchrotron radiation sources enable photoelectron surface analysis of greater resolution. However, synchrotron radiation 
sources are still not widespread and are usually expensive and more complicated than XPS [52].

\subsection{Attenuated total reflection Fourier transform infrared spectroscopy}

Fourier transform infrared spectroscopy is a vibrational spectroscopic technique in which infrared radiation interacts with molecules by exciting their bond vibration modes. Attenuated total reflection (ATR) FTIR is powerful technique suitable for studying surface properties and surface adsorption [53]. When linearly polarised FTIR light travels through an ATR crystal against the sample surface, it is reflected from the crystal/sample interface, creating an evanescent electric field at each reflection. The depth of penetration of the evanescent field is variable, and can be determined using the following formula [53]:

$d_{p}=\frac{\lambda}{2 \pi n_{1} \sqrt{\sin ^{2} \theta-\left(n_{2} / n_{1}\right)^{2}}}$

where $\lambda$ is the wavelength of the light, $n_{1}$ and $n_{2}$ are the refractive index of the ATR crystal and the rare medium, $\theta$ is the angle of incidence. Typical depths of penetration range from microns down to tenths of microns [54].

ATR-FTIR has been widely used for surface characterisation of biomedical materials and protein adsorption [54,55], and more recently to confirm the extent of cross-linking in electrospun chitosan fibres [56]. It can also be used to investigate the degradation [57] and surface conditions of polymer nano-fibrous membranes [21], antigen immobilisation [58] or layer-by-layer (LbL) deposition of coatings of different functionalities on the surface of electrospun polymer membranes.

Due to its larger penetration depth (up to $2 \mu \mathrm{m}$ ), ATR-FTIR is less surface sensitive than XPS or ToFSIMS. However, if these techniques are used complimentarily, they can provide a good range of detailed surface chemistry information [20]. More recently, polarised FTIR has been used to measure the bulk properties of electrospun polymer fibres. The electrical fields and mechanical forces that result from the electrospinning process during the macroscopic nanofibre alignment (i.e., ultilising a rotating mandrel), also align the polymer chains parallel to the direction of fibre alignment [59]. The degree of alignment of the polymer chains can readily be determined through the analysis of polarised FTIR spectra [60]. While the utilisation of this method for the determination of the molecular orientation within electrospun polymer fibres is recent, polarised FTIR is an established and accurate method that has been extensively used to determine the molecular orientation within other polymers. However, more common determinants of the degree of polymer molecular alignment include polarised FTIR coupled with other methods such as polarised Raman spectroscopy and wide angle X-ray diffraction [59].

\subsection{Time-of-flight secondary ion mass spectrometry (ToFSIMS)}

Secondary ion mass spectrometry (SIMS) is a technique with even higher surface sensitivity than XPS, with sampling limited to only the top few atomic layers (typically $1-2 \mathrm{~nm}$ ) $[45,46]$ and a lower detection limit of $0.1 \mathrm{ng} / \mathrm{cm}^{2}$ [46]. ToFSIMS can also be used to create a high-resolution spatial distribution of the location of different chemical components [10,12,13]. Although some advanced XPS and FTIR instruments also have surface mapping functions, these mappings are based on a fixed binding energy or fixed wavenumber making them more inaccurate than those achieved using ToFSIMS.

ToFSIMS uses a beam of energetic (primary) ions, usually argon or gallium, to bombard the surface. As these ions hit the surface, atoms and molecules are removed from the material's surface. A small fraction of the removed atoms and molecules will then be ionised to produce secondary ions, which can be accelerated to a constant kinetic energy and allowed to travel a certain distance before being collected and analysed. The time-of-flight of an ion species in field-free vacuum is related to their individual mass.

Two types of SIMS, static and dynamic, have been developed. In dynamic SIMS the primary ion beam energy is higher than in static SIMS [45], which causes the surface to be eroded by the primary ion beam to reveal the depth chemical profile of the sample. ToFSIMS has been used for characterising the adsorbed protein on surfaces of biomaterials. It can provide composition, conformation, orientation and denaturation information for proteins on material surfaces [61] and has been used to confirm the presence of different amino acids. Since the analysis depth of static SIMS is $10-15 \AA$, the mass spectrum can reveal the amino acid assay of the outer 10-15 $\AA$ of the adsorbed protein. Therefore static SIMS is a powerful technique for the study of the conformation and orientation of proteins.

A disadvantage of SIMS is that it does cause more serious damage to the surface than XPS [62]. Furthermore, the quantification of mass spectra requires standards that should be obtained within the same matrix, and such spectral databases are still being developed. Despite this, it has been successfully used in bulk polymer studies involving poly-L-lysine (PLL) [63] and a poly(vinyl chloride)/poly(methyl methacrylate) (PVC/PMMA) blend [64]. More recently, it has also been successfully applied to electrospun membranes of poly(caprolactone)/cetyltrimethylammonium bromide (PCL/CTAB) [65]. Despite problems with the rough topography, voltage differences and charge build-ups, researchers were able to detect the major diagnostic ions of both the components and roughly predict their proportions, with a small loss in mass accuracy and resolution. Future experiments will hopefully build on this, as the ability of SIMS to examine only the top few layers would be particularly relevant to the fields of tissue engineering where cells interact primarily with these layers. There are no studies as yet involving dynamic SIMS and porous/fibrous polymer samples.

Recently, a modified SIMS technique was used to improve the resolution of components in blended polymeric materials [63]. In blended polymers, the major characteristic ions in one component are often present in the other component, making it impossible to resolve the separate components. This can be overcome if one of the components in the blend is composed of deuterium rather than hydrogen, thus slightly changing the ions formed. This technique may be useful in further studies of blended polymers structures within electrospun membranes if similar problems are encountered.

From the above description of the different analytical techniques available for analysing surfaces, it is apparent that no surface analytical technique provides a full and accurate description of the surface of electrospun membranes. In some circumstances it may be necessary to couple all three major techniques mentioned above to complete the required evaluation of the chemical species on a surface and their distribution. Table 1 offers a summary of the different characterisation techniques.

\section{Surface energy analysis}

The contact angle formed at the junction of three phases (i.e., solid, liquid and gas) reflects the liquid surface tension and the

Table 1

Summary of surface chemical techniques.

\begin{tabular}{|c|c|c|c|c|}
\hline Technique & Depth (m) & $\begin{array}{l}\text { Spatial resolution } \\
(\mu \mathrm{m})\end{array}$ & "Destructivity" & $\begin{array}{l}\text { Sensitivity } \\
\text { (at.\%) }\end{array}$ \\
\hline ATR-FTIR & $2 \times 10^{-6}$ & Down to 15 & Non-destructive & Down to 0.1 \\
\hline XPS & $10 \times 10^{-9}$ & Down to 3 & Non-destructive & Down to 0.01 \\
\hline ToF-SIMS & $2 \times 10^{-9}$ & Down to 0.2 & Destructive & Down to ppm \\
\hline
\end{tabular}


solid surface energy through Young's equation (Eq. (4)) [66]. The contact angle of a liquid on a solid surface is a direct indication of the wettability of the solid by the liquid. It is a sensitive reflection of changes in physicochemical conditions at the surface, and is linked to surface energy and polarity of the outermost surface $(0.3-2 \mathrm{~nm})$ [67].

The surface energy of a solid is determined by the intermolecular forces whose origins are the same as those holding atoms and molecules together in the bulk. An isotropic liquid surface provides a special condition for the surface energy to be directly measured. The surface energy of a pure liquid (which has the unit of $\mathrm{mJ} / \mathrm{m}^{2}$ ) is equivalent to surface tension $(\mathrm{mN} / \mathrm{m})$. However, there is no established method for directly measuring surface energy of a solid surface. The surface energy of the solid can be determined by contact angle methods [67-69] or by gas adsorption methods, including inverse gas chromatography [70] using liquids with known surface energies.

Under optimal conditions, contact angle measurements can sensitively detect molecular and functional group changes on a solid surface. Contact angle methods have been used to monitor LbL deposition of polymers and proteins on surfaces of electrospun membranes, polymer grafting, plasma treatment induced surface polarity and functional group changes [20,71]. However, the contact angle method can only be used on smooth surfaces for surface energy measurements using the Zisman model [72], Fowkes' geometric means model [69], Wu's harmonic means model [73] or the Good-van Oss model [67].

In contrast, an electrospun membrane has a highly porous structure and therefore also has a surface of nano or micro-scale roughness $[9,20]$. Liquid drops on rough and hydrophobic electrospun membranes do not make full contact with the polymer surface (actual contact surface shown in Fig. 4) [74] so contact angle measurements do not correctly reflect the degree of hydrophobicity of the polymer and cannot be used for quantitative or comparison purposes. Zheng et al. showed that while a spin-coated polystyrene film has a contact angle with water of around $93^{\circ}$, the electrospun PS fibre membrane they obtained has a contact angle of $158.1 \pm 2.4^{\circ}$ [75].

There are several contact angle models which allow for rough surfaces, of which the best known are the Wenzel model and the Cassie model. The Wenzel model considers that a liquid drop on

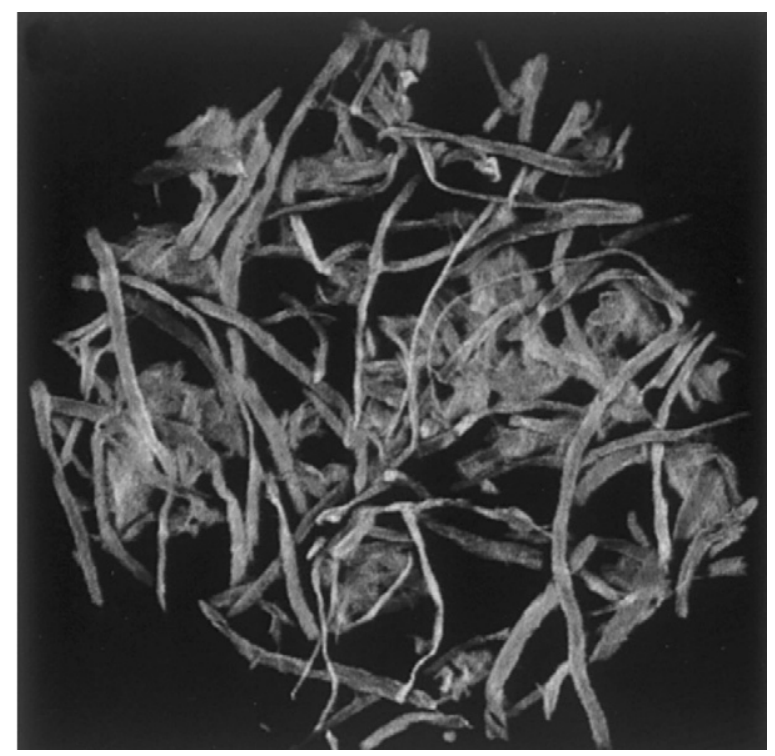

Fig. 4. The actual contact area between a water drop and a paper surface. These images were generated using confocal microscopy. Reproduced from Colloids and Surfaces A with permission from Elsevier [74]. a rough surface has full contact with the surface, i.e., the liquid penetrates into the troughs of the surface texture. This makes it useful in contact angle correction for partially wetting situations. In the Wenzel model, the apparent liquid contact angle on a rough solid surface can be described by the following equation [76]:

$\cos \theta_{\text {rough }}=r \cos \theta_{\text {true }}$

where $r$ is the roughness factor, the ratio of the actual to projected area as expressed below:

$r=\frac{A_{\text {actual }}}{A_{\text {proj }}}$

Nisbet et al. [3] showed the effect of surface roughness on contact angle could be estimated by measuring the solid surface's roughness factor. They used AFM to measure the roughness factors of compression moulded PLGA and PLLA polymer surfaces and predicted the causes of the contact angle changes by various surface treatments.

When measuring contact angle on a hydrophobic electrospun membrane surface, the liquid drop sits on a composite surface comprising of the polymer fibres and air pockets (Fig. 4) [75]. Such a situation is considered in the Cassie's model as follows:

$\cos \theta_{a}=f_{1} \cos \theta_{1}-f_{2}$

where $f_{1}$ is the fraction of the surface that has contact with liquid; $f_{2}$ is the fraction that has no contact with the surface [23]. This model has been widely shown to be reasonably well obeyed on superhydrophobic surfaces $[75,77]$ and hydrophobic surfaces, including hydrophobic non-woven polymer materials. Confocal microscopy has been employed to visually demonstrate the partial contact of a water drop with the surface of a hydrophobised paper surface [74].

Aspler and co-workers provided an alternative technique of greater precision for determining the surface energies [78]. These authors investigated surface energy of fibrous membranes using a series of water-isopropanol solutions, which have different surface tensions. They observed that generally, a solution of isopropanol and water placed on a non-woven surface is imbibed after a certain time delay. However, a solution with a specific isopropanol concentration (and therefore a specific surface tension) placed on the surface of a non-woven sample, is imbibed into the material immediately. The surface tension of this specific solution is an apparent analogy to the critical wetting surface tension of a solid surface [67]. Although this method was originally proposed for characterising the surface energy of paper [78], it was recently employed to characterise surface energies of electrospun membranes [9], and provides a much more reliable measurement of surface energy in an electrospun scaffold than the modified contact angle measurements that were mentioned previously.

\section{Mechanical properties (hardness/modulus testing)}

The mechanical properties of electrospun membranes, in particular the hardness and elastic modulus $(E)$, are often of interest. Nano-indentation is the most common characterisation technique used, chiefly because it allows the hardness of specific areas of an electrospun membrane to be evaluated with very fine spatial resolution and with minimal preparation [78]. However, there are problems with precision and sometimes accuracy when the technique is applied to polymer surfaces, especially fibrous and porous ones. There are now techniques for directly testing the modulus of a single electrospun fibre, which may be of use in some applications [79]. Traditional methods of tensile testing have also been applied to membranes consisting of many fibres, and their use is reviewed here. When choosing a method, the advantages and disadvantages over nano-indentation should be weighed against the properties, 
which are able to be measured, accuracy and time available for preparation and measurement.

\subsection{Nano-indentation}

Nano-indentation has been the preferred method for investigating mechanical properties for many years. While it requires far less preparation than other techniques and can resolve changes in modulus at different points on a sample, previous studies of polymers have encountered many problems in providing reliable modulus measurements. A comprehensive review of the application of nanoindentation to polymers is provided by Van Landingham et al. [78], but its application to nano-fibres will be summarised here, along with a discussion of several new developments.

Many polymers are too soft to be investigated using nanoindentation, while viscoelastic behaviour (creep) and difficulty in accurately characterising tip shape precludes accuracy using the traditional analysis of Oliver and Pharr [80]. Compared with other methods, modulus values of polymers measured by nanoindentation are often much larger or even negative in extreme cases, because of the effects of creep, and it is reasonable to assume that this would also be the case for electrospun polymer fibres. Recent attempts to characterise the creep response of polymeric materials [81] suggest that the modulus depends on the speed of the indentation and thus there is no single well-defined value for modulus in these materials. Modulus values also tend to depend on the depth of indentation, with shallower indentations giving larger moduli [78]. Tip shape has been found to affect the measured values, and calibration of the tip-shape area function seems to be a major problem, with none of the methods used to resolve this producing reproducible results [78]. Rough surfaces of the type observed on electrospun membranes can also act as a "springy", compliant layer, although a method has been proposed to incorporate this into the analysis [82]. There are several different techniques used for the nano-indentation of polymers, which can be related to electrospun membranes. These include depth-sensing indentation (DSI), AFM and interfacial force microscopy (IFM). A detailed discussion of these techniques is outside the focus of this review, hence interested readers are directed to the following research papers $[37,83]$.

Therefore, while nano-indentation is a useful means of testing the mechanical properties of electrospun membranes it is often hard to reproduce and some researchers believe that it has "questionable" links to the real-world behaviour. Having said this, nano-indentation is very useful for testing the relative mechanical properties of membranes within individual laboratories.

\subsection{Dynamic indentation}

Attempts to characterise the viscoelastic behaviour of polymer samples have also been made using dynamic indentation with an oscillating tip. Storage and loss moduli were determined in studies of different polymer materials, which in most cases agreed well with the values determined by dynamic mechanical analysis $[84,85]$. We are not aware of studies involving dynamic indentation on porous surfaces but this method does have potential for characterising storage and loss moduli of electrospun membranes.

\subsection{Problems faced when applying nano-indentation to fibrous membranes}

There are obvious difficulties for determining the moduli of porous samples using nano-indentation. These include ensuring both an ideal contact between tip and fibre and that the fibre is adequately supported to prevent it bending or slipping away from the probe. In one instance membranes were compressed prior to indentation [86], and indentation was then performed on the resulting solid pellet. However, in many instances this technique would have limited value, as generally it is the highly porous nature of electrospun membranes that make them useful in most applications. A more common method is to test a single fibre, which has been spun onto a hard plate [87]. In this situation it was found that the substrate on which the fibre is mounted can affect the measurement if the fibre diameter is below $300 \mathrm{~nm}$. This study also describes problems involving tip contact with a small-radius fibre; the probe may not contact at $90^{\circ}$ or it may slip slightly. The curvature of the fibre should also be considered if this type of analysis is to achieve a more accurate representation of the modulus of the nano-fibre membrane.

While nano-indentation may be a useful and convenient technique for characterising electrospun polymer surfaces, the uncertainties during data analysis must be understood and allowed for, particularly when quoting absolute values of hardness and elastic modulus $[23,24]$. Having said this, nano-indentation experiments have been performed on polymers that have agreed accurately with other testing and accepted values, although as Van Landingham et al. [78] noted, care should be taken when comparing to manufacturer's values, as these can vary greatly with crystallinity and other changeable parameters. New methods of controlling uncertainties would be invaluable, as a simple and accurate nanoindentation method would be a very useful tool for electrospun polymer membrane characterisation.

\section{Mechanical properties-direct methods}

In recent years, several methods have been proposed for the direct testing of the mechanical properties of individual electrospun fibres and the bulk properties of an electrospun matrix. In general these have the advantage of being direct tests of the properties desired, cutting down analysis, and also decreasing the uncertainties in measurements dramatically. Several of these are now discussed.

\subsection{Single fibre testing}

Individual electrospun fibres have been tested both in tensile testing apparatus and by gluing them to an AFM tip [88,89]. Such methods have the advantage of producing an entire force-extension curve, allowing the investigation of other properties such as to yield strength. Smaller diameter fibres generally have a higher modulus, tensile strength and yield stress, but the strain at break is decreased; Fig. 5 shows a typical stress strain curve that highlights the increase in tensile strength and yield stress as fibre diameter decreases. Electrospun fibres are generally strained at a slow rate, in order to allow for viscoelasticity. While this technique is a very reliable means of

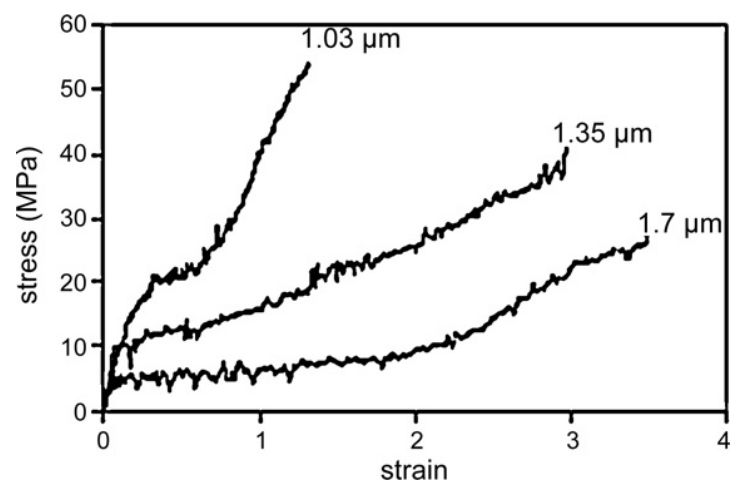

Fig. 5. Stress verses strain behaviour of electrospun PCL microfibres with differing fibre diameters. Both tensile strength and yield stress decreased with increase in fibre diameter [88]. Reproduced from Biomaterials with permission from Elsevier. 


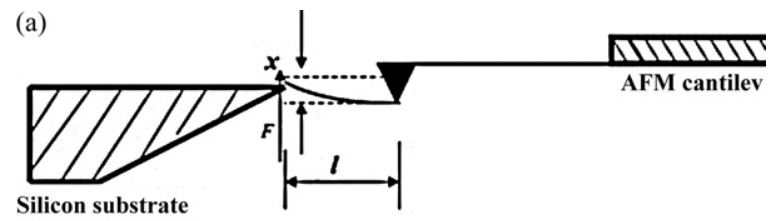

(b)

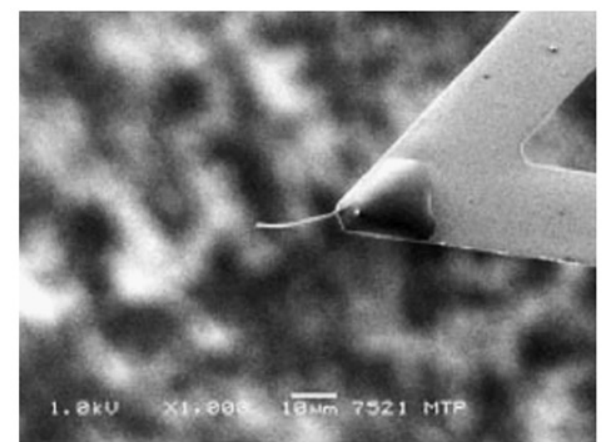

Fig. 6. (A) Schematic of the AFM experimental set-up for conducting the bending test and (B) a polyacrylonitrile fibre attached to the AFM tip [90]. Reproduced from Macromolecular Rapid Communications with permission from Wiley.

determining the mechanical properties of individual electrospun fibres, the process of fibre collection and preparation is very time consuming, especially if multiple fibres are to be tested.

Bending tests have also been performed using an AFM with individual electrospun fibres [82,90] prepared in a similar way to the direct tensile test. The set-up [90] of such a test is shown in Fig. 6. Bending tests only allow the investigation of elastic modulus, and are best for small diameter nano-fibres where shear deformation is negligible [82]. A further extension to this method is to conduct the bend test by suspending individual fibres over a porous surface [91]. This is a way of dispensing with the need to collect individual fibres, as seen in Fig. 6. However, it does require careful searching of the fibre mat in order to find a suitable site for testing which can still be a very time-consuming process.

A new method of investigating the mechanical properties of an electrospun fibre is to employ a cantilever vibration test [92]. Individual fibres are spun between two cantilevers, which are then vibrated to find the resonate frequency. Such a test was designed specifically to allow direct testing of fibres without a timeconsuming collection or searching process, while still achieving modulus measurements in agreement with the bending test of a single electrospun fibre [90]. Cantilever vibration tests only allow for the determination of elastic modulus, but if this is the only bulk mechanical property required to be investigated for a fabri-

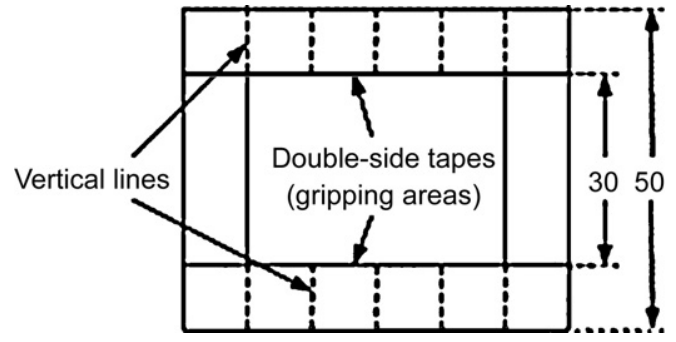

Fig. 7. A paper template proposed to prepare electrospun membranes for bulk tensile testing. Employing such a template can avoid structural damage that occurs during sample mounting for these experiments. Reproduced from Polymer with permission from Elsevier [94].

cated electrospun membrane this technique may be much more convenient than the others described above.

\subsection{Bulk testing of nano-fibre membranes}

It is well understood that the mechanical properties of porous structures can differ markedly from those of the same bulk material. For this reason bulk mechanical tests have also been performed on electrospun membranes [93,94] using a microtensile tester. However, one common problem is that the samples are easily damaged while loading the specimens into such machines; this can be avoided using a protocol similar to that of Huang et al. [94]. Briefly, this involved preparing samples using a paper template that was fabricated in a manner shown in Fig. 7. Double-sided tape was applied to the top and bottom sides of the paper template. The electrospun membrane was then glued to the template and cut along the vertical lines (Fig. 7), with additional single sided tape being applied to where the sample is inserted in to the tensile grips for greater rigidity.

When conducting such tensile tests the strength properties of aligned nano-fibrous matrices depend on the direction of testing $[95,96]$, with increased elastic modulus when tested in a direction parallel to the fibres. Although some studies have reported the absence of such trends [97], this may indicate that in some instances the modulus increase that occurs due to fibre alignment may be influenced by material selection (i.e., the orientation of individual molecules within a specific material, which can be determined using FTIR as discussed above). Tensile testing has been employed to test the bulk properties of electrospun PCL nano-fibre membranes of random and aligned fibre alignment, which successfully characterised the difference in tensile modulus and maximum strength of these different membranes (data shown graphically in
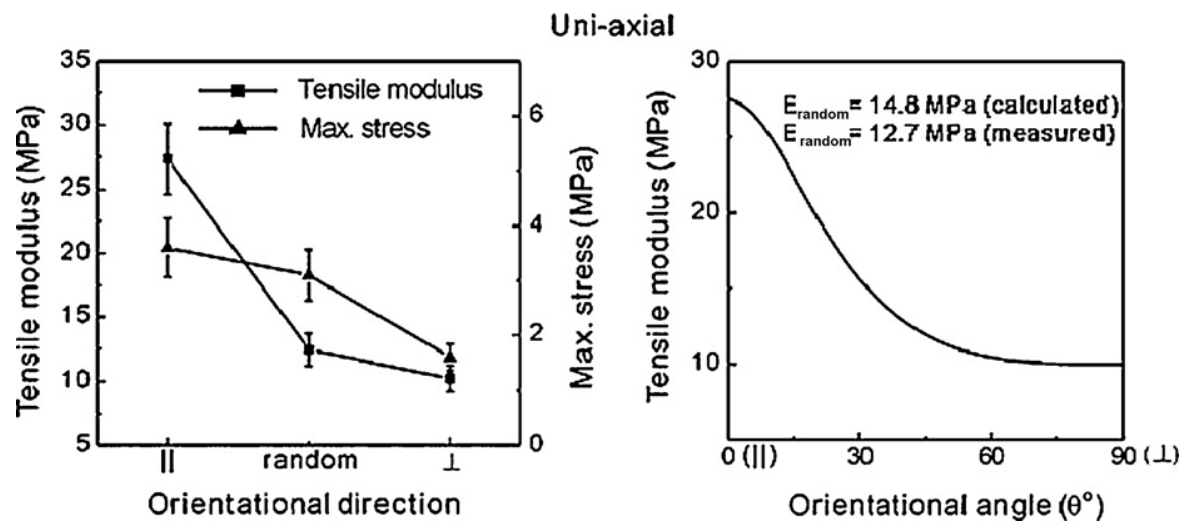

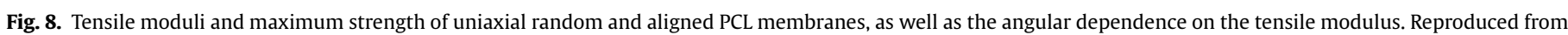
Biomedical Materials with permission from IOP Journals [79]. 

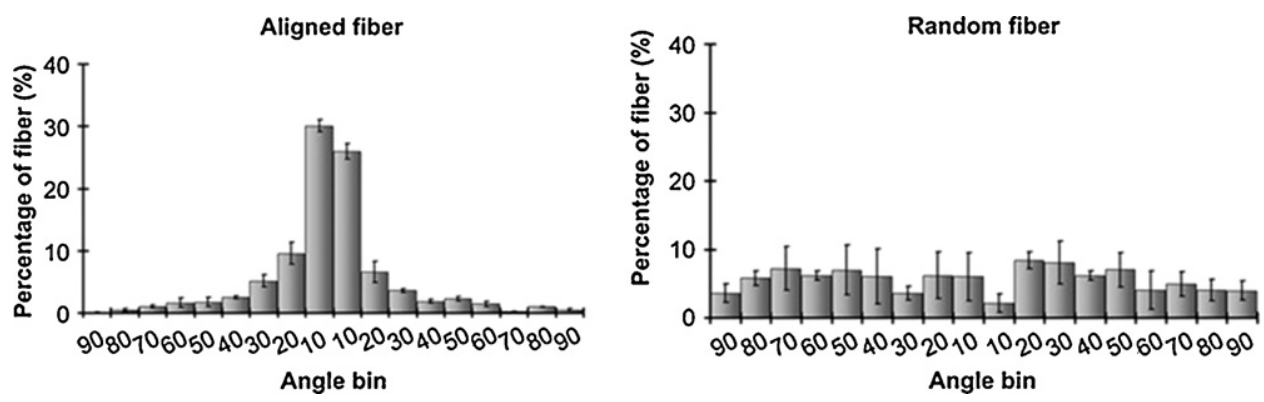

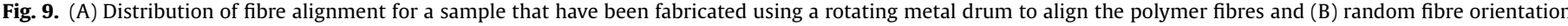
[98]. Reproduced from Biomaterials with permission from Elsevier.

Fig. 8) [79]. In this instance the sample with random fibre alignment had an isotropic tensile modulus of $12.4 \pm 1.3 \mathrm{MPa}$, whereas for the aligned sample the tensile modulus was $27.3 \pm 3.1 \mathrm{MPa}$ parallel to the direction of fibre alignment and 10.2 $\pm 1.2 \mathrm{MPa}$ in the perpendicular direction. The tensile modulus of the membranes was found to increase with the degree of fibre alignment.

PCL fibres have also been strained in tension and have demonstrated a capacity to reorient themselves in the direction of strain, remaining in that orientation after the strain was removed [96]. These observations may explain the difference between strengths in different directions [96]. Strain in the direction of fibre orientation occurred through the strain of individual fibres, while strain in the perpendicular direction was due to the disruption of point bonding between fibres. This development may aid in the interpretation of results from tensile tests, even for randomly oriented fibres. It also shows the importance of the alignment of fibres when placed in a tensile tester.

\section{Alignment characterisation}

It should be noted that when using a rotating mandrel to align nano-fibres, the speed of rotation should be reported as linear speeds $\left(\mathrm{m} \mathrm{s}^{-1}\right)$ rather than angular speeds (RPM), as it is the linear speed of the surface with regard to the fibre that is important in the alignment process. Angular speed is meaningless unless the diameter of the mandrel is also reported, as this will affect the resulting linear speed.

The techniques used to characterise the amount of alignment of the electrospun fibres are mostly based on statistical methods. Strictly speaking, the angle of the fibres is a continuous variable, however most studies [96] have grouped the alignments into discrete subsets and displayed the results as bar charts (Fig. 9) [98]. A continuous distribution may be an alternative choice for modelling the angle of alignment (Fig. 10), and a Gaussian (normal) distribution appears on first sight to be a good fit to the bar charts; however on closer inspection there is a relatively large number of unaligned fibres at the outer edges of the distribution. Statistical tests (Shapiro-Wilks test, using Origin software) on raw alignment data have shown that the data can only be fitted to a normal curve if these unaligned fibres are omitted from the data. This has implications for the use of the standard deviation as a quantifier of the degree of alignment of the nano-fibres, as the standard deviation can be heavily influenced by a very small number of data points lying far outside the normal range. Standard deviation could potentially be very useful in characterising alignment, however effort must be made to account for the outlying data points, as they have an overly strong influence over the standard deviation that is measured.

One technique that avoids this problem to some degree is to measure the percentage of fibres within a given angular range of the principal alignment axis, for example $\pm 15^{\circ}$. This method provides a much simpler characterisation, as it ignores a large population of the fibres, and treats all those within the included range as equivalent. However, this makes the method far more robust and reliable, as it is then the majority of aligned fibres that most affect the final alignment value. This method also prevents comparison between studies, which use different cutoff values, i.e., $\pm 10^{\circ}$ compared with $\pm 15^{\circ}$.

Another alternative method for rapidly measuring the fibre alignment is to employ the two-dimensional fast Fourier transform (FFT) method. This method generates a mathematical frequency domain image from spatial information within an image (typically bright-field or SEM images). Each pixel in the frequency image shows the degree of correspondence between pixels in the original image that have a given distance and direction from each other. Pixel pairs that consistently have similar brightness will appear as bright pixels in the frequency image. The shape of this frequency domain image is ultimately used to determine the degree of fibre alignment, through the formation of an alignment plot [99]. This plot graphs the FFT alignment verses the principle axis of orientation. On such a graph the peak shape and height are characteristic of the degree of alignment within the membrane, with the peak position describing the principle axis of orientation [99]. An example of this process is shown in Fig. 11 where the initial bright-field images are displayed, then the 2D FFT frequency plots and finally the corresponding FFT alignment plots.

Care must be taken when using the FFT method that high quality images are used, or else high frequency noise can mask the useful low frequency information. As the pixels are then radially summed to produce the alignment plot, the plot will be displaced further up the $y$-axis. When this plot is normalised, the resulting alignment value will be under-reported. To avoid this problem, the frequency image should be visually inspected before use to ensure that the high-frequency areas are dark. Another problem is that

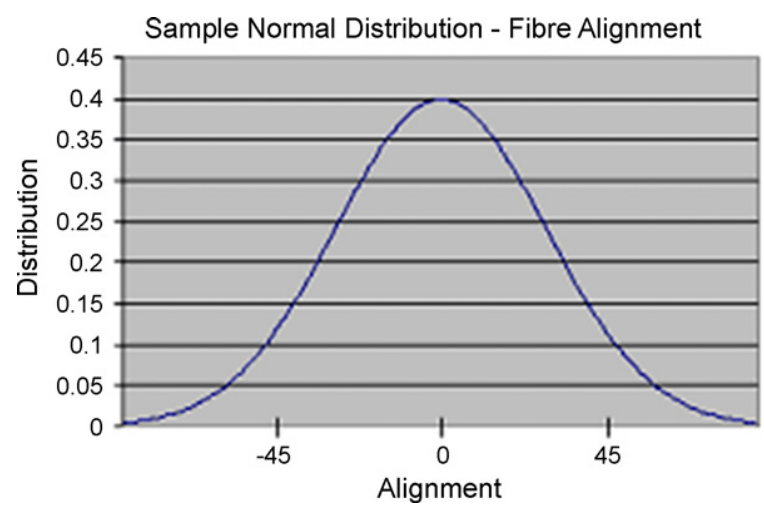

Fig. 10. Author communication of a sample normal (Gaussian) distribution. Probability of a fibre lying between two given angles is the integral of (area under) the curve between those two angles. 

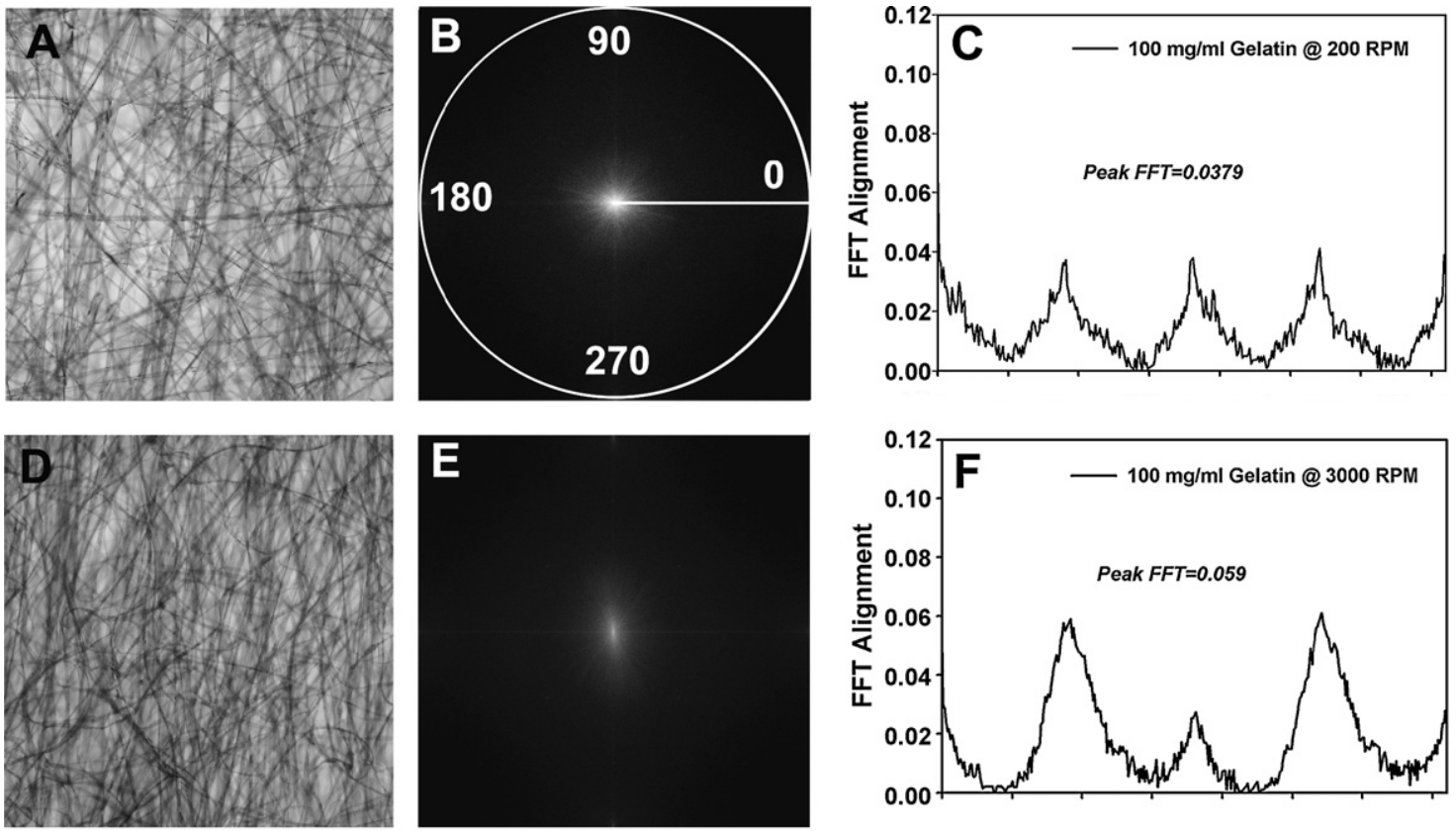

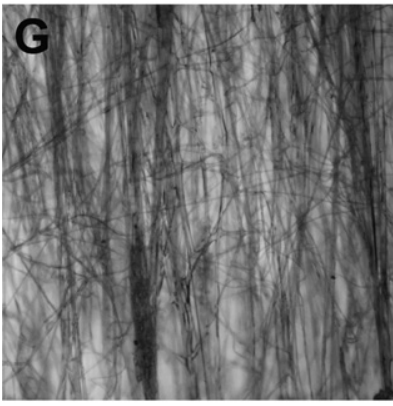

Bright Field Image

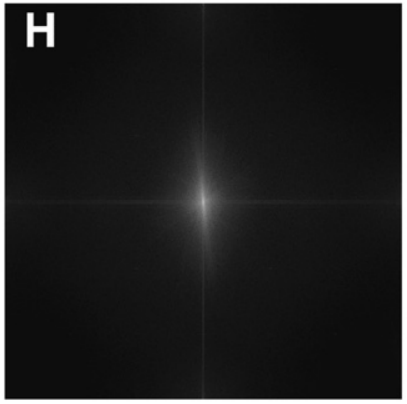

FFT Frequency Image

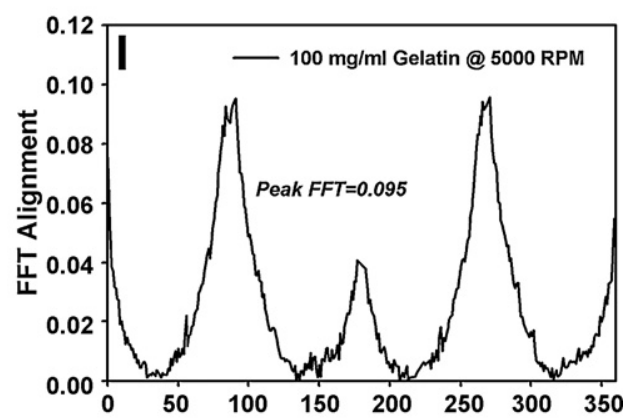

Principle Angle of Orientation

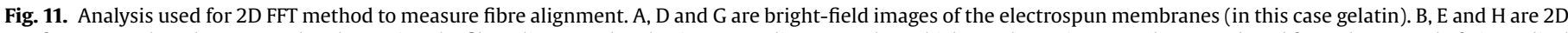

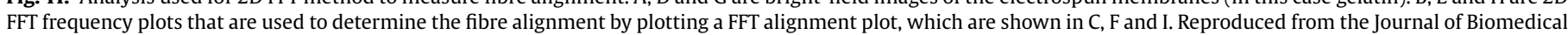
Science-Polymer Edition with permission from BRILL [99].

the reported values appear to have limited resolution; compared to the uncertainty introduced when reading graphs manually, the difference in alignment value between random and highly aligned scaffolds is quite small. Also, it has been noted that uneven illumination within an image can introduce errors. However, the FFT method does have the advantage that it is fully objective; it does not require the researcher to apportion an angle to fibres that are not straight. The interested reader is referred to the following publication that provides an in-depth description of the utilisation of the 2D fast Fourier transformation method for characterising fibre alignment within electrospun membranes [99].

\section{Concluding remarks}

The characterisation of electrospun membranes plays an important role in applying these structures to real-world situations, as the surface and material properties of polymeric membranes strongly influence how they interact with their surrounding environment. There are many important properties when dealing with electrospun interfaces, including the porosity, morphology, surface energy, surface chemical distribution and mechanical strength properties. All these properties influence how an electrospun membrane will interact with the surrounding environment.

There are a vast range of analytical tools at the disposal of surface scientists to characterise these properties. We have addressed the current status in the use of these tools, and touched on possible improvements in their application. Some techniques that have not yet been used for the characterisation of electrospun membranes have also been explored, as we believe that the utilisation of such techniques will dramatically improve the way in which electrospun membranes are currently characterised, and therefore potentially improve their performance in many applications.

\section{Acknowledgements}

We are grateful to the Australian postgraduate award, and the Cooperative Research Centre (CRC) for Polymers (to DRN), the CRC for polymers (to AER), and ARC Discovery (DP0663573) and CRC (to JSF) for funding.

\section{References}

[1] L. Sabbatini, P.G. Zambonin, Surface Characterisation of Advanced Polymers, Weinheim, New York, 1993.

[2] Z.M. Huang, Y.Z. Zhang, M. Kotaki, S. Ramakrishna, Composites Science and Technology 63 (2003) 2223-2253.

[3] D.R. Nisbet, S. Pattanawong, J. Nunan, W. Shen, M.K. Horne, D.I. Finkelstein, J.S. Forsythe, Journal of Colloid and Interface Science 299 (2006) 647-655.

[4] C.Y. Xu, F. Yang, S. Wang, S. Ramakrishna, Journal of Biomedical Materials Research 71 (2004) 154-161.

[5] Z. Ma, M. Kotaki, T. Yong, W. He, S. Ramakrishna, Biomaterials 26 (2005) 2527-2536. 
[6] X. Zong, H. Bien, C. Chung, L. Yin, D. Fang, B. Hsiao, B. Chu, E. Entcheva, Biomaterials 26 (2005) 5330-5338.

[7] W.J. Li, C.T. Laurencin, E.J. Caterson, R.S. Tuan, F.K. Ko, Journal of Biomedical Materials Research 60 (2002) 613-621.

[8] H.L. Schreuder-Gibson, P. Gibson, K. Senecal, M. Sennett, J. Walker, Journal of Advanced Materials 34 (2002) 44-55.

[9] D.R. Nisbet, S. Pattanawong, N.E. Ritchie, W. Shen, D.I. Finkelstein, M.K. Horne, J.S. Forsythe, Journal of Neural Engineering 4 (2007) $1-7$.

[10] B.M. Min, G. Lee, S.H. Kim, Y.S. Nam, T.S. Lee, W.H. Park, Biomaterials 25 (2004) 1289-1297.

[11] H.S. Kim, K.H. Kim, H.J. Jin, I.J. Chin, Macromolecular Symposium 224 (2005) $145-154$.

[12] J.M. Deitzel, W. Kosik, S.H. McKnight, N.C. Beck Tan, J.M. Desimone, S. Crette, Polymer 43 (2002) 1025-1029.

[13] J.E. Sanders, S.E. Lamont, A. Karchin, S.L. Golledge, B.D. Ratner, Biomaterials 26 (2005) 813-818.

[14] A. Alli, B. Hazer, Y. Manceloglu, S. Suzer, European Polymer Journal 42 (2006) 740-750.

[15] J.M. Schakenraad, H.J. Busscher, C.R.H. Wildevuur, J. Arends, Cellular Biophysics 13 (1988) 75-91.

[16] D.R. Absolom, C. Thomson, G. Kruzyk, W. Zingg, A.W. Neumann, Colloids Surface 21 (1986) 447-456.

[17] A. Formhals, US Patent (1934), p. 1,975,504.

[18] B.D. Ratner, Surface Characterisation of Biomaterials, Elsevier, New York, 1998.

[19] M. Bognitzki, W. Czado, T. Frese, A. Schaper, M. Hellwig, M. Steinhart, Advanced Materials 13 (2001) 70-72.

[20] Z. Ma, M. Kotaki, S. Ramakrishna, Journal of Membrane Science 272 (2006) 179-187.

[21] T. Lin, H.G. Wang, H.M. Wang, X. Wang, Nanotechnology 15 (2004) 1375-1381.

[22] D.R. Nisbet, L.M.Y. Yu, T. Zahir, J.S. Forsythe, M.S. Shoichet, Journal of Biomaterials Science Polymer Edition 19 (2008) 623-634.

[23] A.W. Adamson, A.P. Gast, Physical Chemistry of Surfaces, 6th ed., WileyInternational, New York, 1997.

[24] P.A. Webb, C. Orr, Analytical Methods in Fine Particle Technology, Micromeritics Instrument Corporation, USA, 1997.

[25] Y.J. Ryu, H.Y. Kim, K.H. Lee, H.C. Park, D.R. Lee, European Polymer Journal 39 (2003) 1883-1889.

[26] R.E. Dehl, Journal of Biomedical Materials Research 16 (1982) 715-719.

[27] A.K. Jena, K.M. Gupta, Journal of Power Sources 80 (1999) 46-52.

[28] P.V. Grant, C.M. Vaz, P.E. Tomlins, L. Mikhalovska, S. Mikhalovsky, S. James, P. Vadgama, Physical characterization of a polycaprolactone tissue scaffold, in: J.P. Blitz, V.M. Gun'ko (Eds.), Surface Chemistry in Biomedical and Environmental Science, NATO Science Series II: Mathematics, Physics and Chemistry, vol. 228, Springer, 2006, pp. 215-228.

[29] S.J. Gregg, K.S.W. Sing, Adsorption, Surface Area and Porosity, Academic Press, London, 1982.

[30] S.C. Baker, N. Atkin, P.A. Gunning, N. Granville, K. Wilson, D. Wilson, J. Southgate, Biomaterials 27 (2006) 3136-3146.

[31] R.R. Rye, J.A. Mann, F.G. Yost, Langmuir 12 (1996) 555-565.

[32] Y. Srivastava, I. Loscertales, M. Marquez, T. Thorsen, Microfluid Nanofluid 4 (2008) 245-250.

[33] J. Yuh, J.C. Nino, W.M. Sigmund, Materials Letters 59 (2005) 3645-3647.

[34] F.K.Y. Gogotsi, A. Ali, N. Naguib, H. Ye, G.L. Yang, C. Li, P. Willis, Advanced Materials 15 (2003) 1161-1165.

[35] R. García, R. Pérez, Surface Science Reports 47 (2002) 197-301.

[36] C.T. Lim, E.P.S. Tan, S.Y. Ng, Applied Physics Letters 92 (2008) 141908.

[37] B. Bhusan, H. Fuchs, S. Hosaka (Eds.), Applied Scanning Probe Methods, Springer, 2004.

[38] J.F. Lübben, G. Fortunato, M. Halbeisen, S. Houis, M. Keller, E. Körner, Journal of Physics: Conference Series 61 (2007) 735-739.

[39] S. Borhani, S.A. Hosseini, S.G. Etemad, J. Militký, Journal of Applied Polymer Science 108 (2008) 2994-3000.

[40] K.-H. Jung, M.-W. Huh, W. Meng, J. Yuan, S.H. Hyun, J.-S. Bae, S.M. Hudson, I.-K. Kang, Journal of Applied Polymer Science 105 (2007) 2816-2823.

[41] A. Hammiche, H. Pollock, M. Reading, M. Claybourn, P. Turner, K. Jewkes, Applied Spectroscopy 53 (1999) 810.

[42] I. Koprinarov, A. Hitchcock, W. Li, Y. Heng, H. Stoever, Macromolecules 34 (2001) 4424.

[43] R. Jaeger, H. SchOnherr, G.J. Vancso, Macromolecules 29 (1996) 7634-7636.

[44] G. Beamson, D. Briggs, High Resolution Xps of Organic Polymers, John Wiely \& Sons, Chichester, England, 1992.

[45] H. Liu, T.J. Webster, Biomaterials 28 (2007) 354-369.

[46] M.S. Wagner, S.L. McArthur, M. Shen, T.A. Horbett, D.G. Castner, Journal of Biomaterials Science Polymer Edition 13 (2002) 407-428.

[47] K. Artyushkova, J.E. Fulghum, Journal of Electron Spectroscopy and Related Phenomena 121 (2001) 33-55.

[48] P. Rossini, P. Colpo, G. Ceccone, K.D. Jandtb, F. Rossia, Materials Science and Engineering 23 (2003) 353-358.

[49] Y.Z. Zhang, X. Wang, Y. Feng, J. Li, C.T. Lim, S. Ramakrishna, Biomacromolecules 7 (2006) 1049-1057.

[50] P.Zhao, H.Jiang, H. Pan, K.Zhu, W. Chen, Journal of Biomedical Material Research A 83 (2007) 372-382.

[51] W. He, Z.W. Ma, T. Yong, W.E. Teo, S. Ramakrishna, Biomaterials 26 (2005) 7606-7615

[52] J.B. Wachtman, Z.H. Kalman, Characterization of Materials, ButterworthHeinemann, Greenwich, Manning, 1993, pp. 200-220.
[53] A.R. Hind, S.K. Bhargava, A. Mckinnon, Advanced Colloid and Interface Science 93 (2001) 91-114.

[54] K.K. Chittur, Biomaterials 19 (1998) 357-369.

[55] R.M. Cornelius, P.W. Wojciechowski, J.L. Brash, Journal of Colloid and Interface Science 150 (1992) 121-133.

[56] J.D. Schiffman, C.L. Schauer, Biomacromolecules 8 (2007) 594-601.

[57] X.L. Deng, G. Sui, M.L. Zhao, G.Q. Chen, X.O. Yang, Journal of Biomaterials Science Polymer Edition 18 (2007) 117-130.

[58] Q. Shi, X. Chen, T. Lu, X. Jing, Biomaterials 29 (2008) 1118-1126.

[59] M.V. Kakade, S. Givens, K. Gardner, K.H. Lee, D.B. Chase, J.F. Rabolt, Journal of the American Chemical Society 129 (2007) 2777-2782.

[60] K.H. Lee, K.W. Kim, A. Pesapane, H.Y. Kim, J.F. Rabolt, Macromolecules 41 (2008) 1494-1498.

[61] C.D. Tidwell, D.G. Castner, S.L. Golledge, B.D. Ratner, K. Meyer, B. Hagenhoff, A Benninghoven, Surface and Interface Analysis 31 (2001) 724-733.

[62] T. Takahagi, Y. Nakayama, F. Soeda, A. Ishitani, Journal of Applied Polymer Science 41 (1990) 1451-1458.

[63] S.K. Tam, J. Dusseault, S. Polizu, M. Ménard, J.-P. Hallé, L.H. Yahia, Biomaterials 26 (2005) 6950-6961.

[64] K. Norrman, K.B. Haugshøj, N.B. Larsen, Journal of Physical Chemistry B 106 (2002) 13114-13121.

[65] P.V. Royen, E. Schacht, L. Ruys, L.V. Vaeck, Rapid Communication Mass Spectrometry 20 (2006) 346-352.

[66] P.C. Hiemenz, R. Rajogopalan, Principles of Colloid and Surface Chemistry, 3rd ed., Marcel Dekker, New York, 1997.

[67] R.J. Good, Contact angle, wetting and adhesion: a critical review, in: Contact Angle, Wettability and Adhesion, Utrecht, Netherlands, 1993, pp. 3-36.

[68] D.K. Owens, R.C.J. Wendt, Journal of Applied Polymer Science 13 (1969) 1741.

[69] F.M. Fowkes, M.A. Mostafa, Industrial Engineering in Chemical Production Research and Development 17 (1978) 3.

[70] A. Voelkelk, Critical Review of Analytical Chemistry 22 (1991) 411-439.

[71] Q.F. Wei, W.D. Gao, D.Y. Hou, X.Q. Wang, Applied Surface Science 245 (2005) $16-20$.

[72] W.A. Zisman, ACS Series 43 (1964) 1.

[73] S. Wu, Polymer Interface and Adhesion, Marcel Dekker, New York, 1982, p. 178

[74] W. Shen, Y. Filonenko, Y. Truong, N. Brack, P. Pigram, I.H. Parker, J. Liesegang, Colloids and Surfaces A Physicochemical and Engineering Aspects 173 (2000) 117-126.

[75] J. Zheng, A. He, J. Li, J. Xu, C.C. Han, Polymer 47 (2006) 7095-7102.

[76] R.N. Wenzel, Industrial Engineering Chemistry 28 (1936) 994-998.

[77] E. Puukilainen, T. Rasilainen, M. Suvanto, T.A. Pakkanen, Langmuir 23 (2007) 7263-7268.

[78] M.R. Van Landingham, J.S. Villarubia, W.F. Guthrie, G.F. Meyers, Macromolecular Symposia 167 (2001) 15-43.

[79] G.H. Kim, Biomedical Materials 3 (2008), doi:10.1088/1748-6041/1083/1082/ 025010.

[80] W.C. Oliver, G.M. Pharr, Journal of Materials Research 7 (June (6)) (1992) 1564-1580.

[81] M.L. Oyen, Acta Materialia 55 (2007) 3633-3639.

[82] E.P.S. Tan, C.T. Lim, Nanomechanical testing of polymeric nanofibers, in: C. Quan, F.S. Chau, A. Asundi, B.S. Wong, C.T. Lim (Eds.), Third International Conference on Experimental Mechanics and Third Conference of the Asian Committee on Experimental Mechanics. Proceedings of SPIE, Singapore, 2004, pp. 849-855.

[83] M.R. Van Landingham, J.S. Villarubia, W.F. Guthrie, G.F. Meyers, Macromolecular Syposia 167 (2001) 15-45.

[84] G.M. Odegard, T.S. Gates, H.M. Herring, Experimental Mechanics 45 (2005) 130-136.

[85] C.C. White, M.R. Van Landingham, P.L. Drzal, N.-K. Chang, S.-H. Chang, Journal of Polymer Science: Part B: Polymer Physics 43 (2005) 1812-1824.

[86] S.A. Catledge, W.C. Clem, N. Shrikishen, S. Chowdhury, A.V. Stanishevsky, M Koopman, Y.K. Vohra, Biomedical Materials 2 (2007) 142-150.

[87] E.P.S. Tan, C.T. Lim, Applied Physics Letters 87 (2005) 123106.

[88] E.P.S. Tan, S.Y. Ng, C.T. Lim, Biomaterials 26 (2005) 1453-1456

[89] E.P.S. Tan, C.N. Goh, C.H. Sow, C.T. Lim, Applied Physics Letters 86 (2005) 073115

[90] S.-Y. Gu, Q.-L. Wu, J. Ren, G.J. Vancso, Macromolecular Rapid Communication 26 (2005) 716-720.

[91] S.-H. Lee, C. Tekmen, W.M. Sigmund, Materials Science and Engineering A 398 (2005) 77-81.

[92] P.A. Yuya, Y. Wen, J.A. Turner, Y.A. Dzenis, Z. Li, Applied Physics Letters 90 (2007) 111909.

[93] W. He, T. Yong, Z.W. Ma, R. Inai, W.E. Teo, S. Ramakrishna, Tissue Engineering 12 (2006) 2457-2466.

[94] Z.-M. Huang, Y.Z. Zhang, S. Ramakrishna, C.T. Lim, Polymer 45 (2004) 5361-5368.

[95] V. Thomas, M.V. Jose, S. Chowdury, J.F. Sullivan, D.R. Dean, Y.K. Vohra, Journa of Biomaterial Science Polymer Edition 17 (2006) 969-984.

[96] J. Johnson, A. Ghosh, J. Lannutti, Journal of Applied Polymer Science 104 (2007) 2919-2927.

[97] C. Ayres, G. Bowlin, S. Henderson, L. Taylor, J. Shultz, J. Alexander, T. Telemeco, D. Simpson, Biomaterials 27 (2006) 5524.

[98] Y.T. Kim, V.K. Haftel, S. Kumar, R.V. Bellamkonda, Biomaterials 29 (2008) 3117-3127.

[99] C. Ayres, B.S. Jha, H. Meredith, J.R. Bowman, G.L. Bowlin, S.C. Henderson, D.G. Simpson, Journal of Biomedical Science Polymer Edition 19 (2008) 603-621. 